A report on ongoing and planned non-pharmacological intervention studies for the treatment and prevention of malnutrition in elderly a MaNuEL report

\title{
MaNuEL
}

JPI Knowledge Hub Malnutrition in the Elderly

published in Kiel (September 2018)

https://dx.doi.org/10.21941/MaNuELworkpackage42

Geisler, Corinna

Institute of Human Nutrition, Christian-Albrechts-University Kiel, Germany

Correspondence author: cgeisler@nutrition.uni-kiel.de 


\section{A report on ongoing and planned non-pharmacological intervention studies for the treatment and prevention of malnutrition in elderly a MaNuEL report}

The "Malnutrition in the Elderly Knowledge Hub" (MaNuEL) is an action program as part of the Strategic Research Agenda of the Joint Programming Initiative "A Healthy Diet for a Healthy Life". In the MaNuEL project experts of 22 research groups from 7 countries (Austria, France, Germany, Ireland, Spain, the Netherlands and New Zealand) came together to bundle up all the knowledge on malnutrition.

MaNuEL started on March 1, 2016 and has obtained funding for two years. For further information, please follow https://www.healthydietforhealthylife.eu/index.php/joint-actions/malnutrition.

MaNuEL is built on five interconnected malnutrition work packages. All packages cover different domains which are important to improve the knowledge/awareness about malnutrition in older persons e.g. definition, optimal screening, and optimal prevention and treatment, as well as implementation of evidence-based knowledge in clinical practice, policy and education.

One of these work packages is the work package (WP) with the title: Prevention and treatment of malnutrition in older persons (WP 4).

\section{WP 4: Prevention and treatment of malnutrition in older persons.}

The subject of WP 4 is the prevention and treatment of malnutrition in older adults. Accordingly, we drafted an overview about ongoing and planned non-pharmacological intervention studies for malnutrition worldwide. Thus, the aim of subtask 4.2 was to search for ongoing and planned nonpharmacological intervention studies.

Main inclusion criteria:

1. non-pharmacological intervention studies

2. nutritional problems and malnutrition

3. in healthy older people

4. study had not yet been completed

Main search topics:

1. >60 years and healthy

2. underweight, unattended weight loss

3. malnutrition and/or energy-protein malnutrition

4. energy and/or protein supply

5. additional interventions

Exclusion criterion: pharmacological interventions patients

Search strategies are listed in table 1 on the next page. 


\section{Table 1 Search strategies}

\begin{tabular}{|c|c|c|}
\hline clinical register & search strategies & comments/results \\
\hline \multirow[t]{12}{*}{ EU clinical trials register } & & free choice of tags \\
\hline & protein energy malnutrition & \\
\hline & protein or energy and malnutrition & \\
\hline & protein or energy or malnutrition & \\
\hline & under weight or underweight or weight loss & \\
\hline & under weight protein & results patients only \\
\hline & underweight protein & \\
\hline & under weight energy & results patients only \\
\hline & $\begin{array}{l}\text { under weight or underweight or weight loss or weight loss or under fed or underfed or protein } \\
\text { energy malnutrition or protein calorie malnutrition or undernourish or under nour or under nutr } \\
\text { or undernutr or maln or malnutrition }\end{array}$ & \\
\hline & protein energy malnutrition and healthy & \\
\hline & consmeer & \\
\hline & risk of malnutrition & $\begin{array}{l}\text { EudraCT Number:2007- } \\
\text { 006153-31 }\end{array}$ \\
\hline \multicolumn{3}{|l|}{ clinicaltrials.gov } \\
\hline & $\begin{array}{l}\text { malnutrition | Open Studies | Interventional Studies | protein | Senior | Studies that accept } \\
\text { healthy volunteers }\end{array}$ & NCT02683720 \\
\hline & $\begin{array}{l}\text { Open Studies | Interventional Studies | malnutrition | protein, energy | Senior | Studies that } \\
\text { accept healthy volunteers }\end{array}$ & \\
\hline & $\begin{array}{l}\text { under weight or underweight or weight loss | Open Studies | Interventional Studies | } \\
\text { malnutrition | protein, energy | Senior | Studies that accept healthy volunteers }\end{array}$ & \\
\hline & weight loss & \\
\hline & underweight & \\
\hline & $\begin{array}{l}\text { low body weight | Open Studies | Interventional Studies | protein | Senior | Studies that accept } \\
\text { healthy volunteers }\end{array}$ & NCT02912130 \\
\hline & $\begin{array}{l}\text { malnutrition | Open Studies | Interventional Studies | energy | Senior | Studies that accept } \\
\text { healthy volunteers }\end{array}$ & NCT03032237 \\
\hline & $\begin{array}{l}\text { malnutrition | Open Studies | Interventional Studies | protein | Senior | Studies that accept } \\
\text { healthy volunteers }\end{array}$ & \\
\hline
\end{tabular}




\begin{tabular}{|c|c|c|}
\hline & $\begin{array}{l}\text { undernourished; Open Studies; Interventional Studies; protein; Senior; Studies that accept } \\
\text { healthy volunteers }\end{array}$ & \\
\hline & $\begin{array}{l}\text { protein energy malnutrition | Open Studies | Interventional Studies | Senior | Studies that } \\
\text { accept healthy volunteers }\end{array}$ & NCT03032237 (s. a.) \\
\hline & $\begin{array}{l}\text { protein calorie malnutrition | Open Studies | Interventional Studies | Senior | Studies that } \\
\text { accept healthy volunteers }\end{array}$ & \\
\hline & $\begin{array}{l}\text { under nour | Open Studies | Interventional Studies | protein | Senior | Studies that accept } \\
\text { healthy volunteers }\end{array}$ & \\
\hline & $\begin{array}{l}\text { under nour | Open Studies | Interventional Studies | energy | Senior | Studies that accept } \\
\text { healthy volunteers }\end{array}$ & \\
\hline & $\begin{array}{l}\text { under weight or underweight or weight loss or weight loss (MeSH Terms) or under fed or } \\
\text { underfed or protein energy malnutrition or protein energy malnutrition (MeSH Terms) or } \\
\text { protein calorie malnutrition or undernourish or under nour or under nutr or undernutr or maln } \\
\text { or malnutrition (MeSH Terms) not child }\end{array}$ & \\
\hline & $\begin{array}{l}\text { risk of malnutrition | Recruiting, Not yet recruiting, Active, not recruiting, Enrolling by invitation } \\
\text { Studies | Interventional Studies | Senior }\end{array}$ & $\begin{array}{l}\text { NCT01057914; } \\
\text { NCT02857049; } \\
\text { NCT03459001; } \\
\text { NCT03417531; } \\
\text { NCT03412695; } \\
\text { NCT03402061; } \\
\text { NCT03073811; } \\
\text { NCT01057914 }\end{array}$ \\
\hline & $\begin{array}{l}\text { risk of malnutrition | Recruiting, Not yet recruiting, Active, not recruiting, Enrolling by invitation } \\
\text { Studies | Senior | Studies that accept healthy volunteers }\end{array}$ & \\
\hline & $\begin{array}{l}\text { risk of malnutrition | Recruiting, Not yet recruiting, Active, not recruiting, Enrolling by invitation } \\
\text { Studies | Interventional Studies | Senior | Studies that accept healthy volunteers }\end{array}$ & \\
\hline & $\begin{array}{l}\text { Recruiting, Not yet recruiting, Active, not recruiting, Enrolling by invitation Studies | } \\
\text { Interventional Studies | Leucine | Senior | Studies that accept healthy volunteers }\end{array}$ & $\begin{array}{l}\text { NCT03197402; } \\
\text { NCT03281434; } \\
\text { NCT02918981; } \\
\text { NCT03299972 }\end{array}$ \\
\hline & Interventional Studies | Leucine | Senior | Studies that accept healthy volunteers & \\
\hline cc.nih.gov/gov/home/clinicalstudies & & currently not available \\
\hline & protein, healthy & \\
\hline & protein, energy, healthy & \\
\hline & protein, energy & \\
\hline & protein & \\
\hline & malnutrition & \\
\hline
\end{tabular}




\begin{tabular}{|c|c|c|}
\hline & risk of malnutrition and senior and leucine and intervention & \\
\hline \multicolumn{3}{|l|}{ researchmatch.org/trials/ } \\
\hline & intervention, 65 , healthy, elderly & \\
\hline & intervention, 65 , healthy, protein & \\
\hline & intervention, 65 , healthy, energy & \\
\hline & risk of malnutrition, healthy, $60+$, intervention & \\
\hline \multicolumn{3}{|c|}{ drks-neu.uniklinik-freiburg.de } \\
\hline & malnutrition, Diät, Nahrungsergänzung und and. Studienthemen & \\
\hline & malnutrition, protein, Diät, Nahrungsergänzung und and. Studienthemen & \\
\hline & risk of malnutrition, $>65$ Jahre, Interventionell, Diät, Nahrungsergänzungsmittel & \\
\hline & Leucine, Leucin, Malnutrition, > 65 Jahre, Interventionell, Diät, Nahrungsergänzungsmittel & \\
\hline \multicolumn{3}{|l|}{ isrctn } \\
\hline & ongoing, nutritional, metabolic, endocrine & \\
\hline & $\begin{array}{l}\text { malnutrition or mal nutrition or undernourished AND ( Condition: healthy elderly AND } \\
\text { Interventions: energy, protein AND Participant age range: Senior ) }\end{array}$ & \\
\hline & $\begin{array}{l}\text { malnutrition AND ( Condition: healthy elderly AND Interventions: energy or protein AND } \\
\text { Recruitment Status: Recruiting ) }\end{array}$ & \\
\hline & $\begin{array}{l}\text { malnutrition ongoing, nutritional, metabolic, endocrine AND ( Condition: healthy elderly AND } \\
\text { Interventions: energy or protein AND Recruitment Status: Recruiting ) }\end{array}$ & \\
\hline & $\begin{array}{l}\text { nutrition AND ( Condition: healthy elderly AND Interventions: energy or protein AND } \\
\text { Recruitment Status: Recruiting ) }\end{array}$ & \\
\hline & recruiting, senior, healthy interventions: leucine, gender: both & \\
\hline & risk of malnutrition, Senior, gender: both, & \\
\hline & leucine & ISRCTN12419961 \\
\hline eudract.ema.europa.eu & & Just registration tool \\
\hline \multicolumn{3}{|c|}{ gsk-clinicalstudyregister.com } \\
\hline & nutrition protein energy, interventional, recruiting, senior elderly & \\
\hline & malnourished or undernourished or underfed, interventional, recruiting, elderly & \\
\hline & healthy, interventional, recruiting,elderly & \\
\hline & healthy, dietary supplements, interventional, recruiting,elderly & \\
\hline
\end{tabular}


risk of malnutrition, interventional, gender: both, Age: 60-100 years

\section{WHO International clinical trials registry}

platform

anzctr.org

nutrition or malnourished and elderly

nutrition elderly, young, dietary protein intake, recruiting

NTR6038

health of the elderly

health promotion

risk of malnutriton

weight loss; energy; prevention, Lifestyle, behavior; interventional; healthy volunteers; not yet

recruiting, recruiting, active not recruiting;

weight loss; energy; prevention, Lifestyle, behavior, treatment: drugs; interventional; healthy

volunteers; condition category: diet and nutrition; not yet recruiting, recruiting, active not

recruiting;

weight loss, underweight, malnutrition; energy, free amino acids, protein; prevention,

Lifestyle, behavior, treatment: drugs; interventional; healthy volunteers; condition category:

diet and nutrition; not yet recruiting, recruiting, active not recruiting;

weight loss, underweight, malnutrition; energy, free amino acids, protein; prevention,

Lifestyle, behavior; treatment: drugs; interventional; healthy volunteers; condition category:

public health; not yet recruiting, recruiting, active not recruiting;

healthy patient studies

body composition trials

diet and nutrition

weight loss

weight loss clinical trials, senior oder $>60$ years

leucine

risk of malnutrition in elderly

\section{ACTRN12615001134516}


Table 2 presented the results of different search strategies. An example of a search is mentioned below:

\section{Search terms in ClinicalTrials.gov:}

- Condition or disease: malnutrition

- open studies

- study type: interventional studies

- Intervention/treatment: protein

- Age Group: senior (66+)

- studies that accept healthy volunteers

2. Results: 5 studies

3. Selected study:

Increase Protein Intake of Older Meal Service Clients with Readymade Protein-rich Meals and Foods (NCT03032237; ConsuMEER onderzoek) 


\section{Table 2 Selected studies}

\begin{tabular}{|c|c|c|c|c|c|c|c|c|c|}
\hline No. & Register & Study title & $\begin{array}{l}\text { Date of } \\
\text { submission }\end{array}$ & ID-Nr. & Country & $\mathrm{n}$ & Age & Inclusion criteria & Intervention \\
\hline 1. & $\begin{array}{l}\text { Clinical } \\
\text { Trials.gov }\end{array}$ & $\begin{array}{l}\text { Evaluating the Efficacy of a Novel Oral } \\
\text { Supplement in Tackling Malnutrition in } \\
\text { the Elderly (ProMO) }\end{array}$ & $02 / 12 / 2016$ & NCT02683720 & Netherlands & 82 & $\begin{array}{l}65 \text { years } \\
\text { and older }\end{array}$ & $\begin{array}{l}\text { age } 65+\text {; at risk of } \\
\text { malnutrition/malnourished }\end{array}$ & Dietary Supplement: ONS \\
\hline 2. & $\begin{array}{l}\text { Clinical } \\
\text { Trials.gov }\end{array}$ & $\begin{array}{l}\text { The Multidimensional Effects of Exercise } \\
\text { and Nutritional Interventions on } \\
\text { Musculoskeletal Functioning, Nutritional } \\
\text { Status and Quality of Life in Age-related } \\
\text { Sarcopenia }\end{array}$ & $09 / 12 / 2016$ & NCT02912130 & $\begin{array}{l}\text { UK - Liverpool } \\
\text { Hope University }\end{array}$ & $\begin{array}{l}\text { Estima- } \\
\text { ted } \\
\text { Enroll- } \\
\text { ment } \\
160\end{array}$ & $\begin{array}{l}60-90 \\
\text { years }\end{array}$ & $\begin{array}{l}\text { Male and Female participants; } 60 \text { - } 90 \\
\text { years of age; Resident in North West, } \\
\text { England, UK; BMI } 18,5-30 \mathrm{~kg} / \mathrm{m} 2 ; \text { can } \\
\text { speak and understand English; willing } \\
\text { to consent and follow the study } \\
\text { procedures }\end{array}$ & $\begin{array}{l}\text { Protein Supplementation } \\
\text { i.e. } 1.2-1.5 \mathrm{~g} / \mathrm{kg} \text { body weight } \\
\text { per day }\end{array}$ \\
\hline 3. & $\begin{array}{l}\text { Clinical } \\
\text { Trials.gov }\end{array}$ & $\begin{array}{l}\text { Testing the Effectiveness and Acceptance } \\
\text { of Home-delivered Protein-rich Meals } \\
\text { and Foods on the Intake of Community- } \\
\text { dwelling Older Adults }\end{array}$ & $01 / 16 / 2017$ & NCT03032237 & $\begin{array}{l}\text { Netherlands - } \\
\text { HAN University } \\
\text { of Applied } \\
\text { Sciences } \\
\text { (FrieslandCam- } \\
\text { pina Germany } \\
\text { GmbH) }\end{array}$ & $\begin{array}{l}\text { Estima- } \\
\text { ted } \\
\text { Enroll- } \\
\text { ment } \\
100\end{array}$ & $\begin{array}{l}65 \text { years } \\
\text { and older }\end{array}$ & $\begin{array}{l}\text { Aged } 65 \text { years or over; living at home; } \\
\text { be a client of maaltijdservice.nl; being } \\
\text { able to eat by themselves; have a } \\
\text { microwave to heat meals; live in } \\
\text { Nijmegen or Den Bosch area; being } \\
\text { able to understand, read and speak } \\
\text { Dutch; having signed informed } \\
\text { consent }\end{array}$ & $\begin{array}{l}\text { Protein-rich readymade } \\
\text { meals and protein-rich } \\
\text { dairy products }-1.2 \mathrm{~g} / \mathrm{kg} \\
\text { body weight } / \mathrm{d}\end{array}$ \\
\hline
\end{tabular}




\begin{tabular}{|c|c|c|c|c|c|c|c|c|c|}
\hline No. & Register & Study title & $\begin{array}{l}\text { Date of } \\
\text { submission }\end{array}$ & ID-Nr. & Country & $\mathrm{n}$ & Age & Inclusion criteria & Intervention \\
\hline 4. & $\begin{array}{l}\text { Clinical } \\
\text { Trials.gov }\end{array}$ & $\begin{array}{l}\text { Effects of Six Months Treatment With } \\
\text { Nutritional Supplements or Dietary Advice, } \\
\text { Alone or in Combination on Mortality, } \\
\text { Health-related Quality of Life and Health-care } \\
\text { Consumption for Elderly Patients at Risk of } \\
\text { Malnutrition }\end{array}$ & $01 / 27 / 2010$ & NCT01057914 & $\begin{array}{l}\text { Sweden, } \\
\text { Uppsala } \\
\text { University }\end{array}$ & 800 & $\begin{array}{l}65 \text { years } \\
\text { and older }\end{array}$ & $\begin{array}{l}\geq 65 \text { years old; Admitted to one of } \\
\text { the participating wards during the } \\
\text { recruitment period }\end{array}$ & $\begin{array}{l}\text { 1. Dietary supplement: } \\
\text { Nutritional } \\
\text { supplementation with a } \\
\text { liquid formula } \\
\text { The patient consumes } 400 \\
\text { kcal from a liquid } \\
\text { supplementation formula } \\
\text { 2. Other: Dietary advice } \\
\text { The patient receives } \\
\text { dietary advice for one hour } \\
\text { during the hospital stay } \\
\text { 3. Other: Combination of } \\
\text { supplement and dietary } \\
\text { advice } \\
\text { The patient receives } \\
\text { dietary advice for one hour } \\
\text { during the hospital stay } \\
\text { and consumes } 400 \text { kcal } \\
\text { from liquid } \\
\text { supplementation formula } \\
\text { for six months after } \\
\text { discharge }\end{array}$ \\
\hline
\end{tabular}




\begin{tabular}{|c|c|c|c|c|c|c|c|c|c|}
\hline No. & Register & Study title & $\begin{array}{l}\text { Date of } \\
\text { submission }\end{array}$ & ID-Nr. & Country & $\mathrm{n}$ & Age & Inclusion criteria & Intervention \\
\hline 5. & $\begin{array}{l}\text { Clinical } \\
\text { Trials.gov }\end{array}$ & $\begin{array}{l}\text { Effective Consumption of Oral Nutritional } \\
\text { Supplements in Geriatrics: A Study } \\
\text { Evaluating a Strategy Based on a } \\
\text { Preliminary Degustation }\end{array}$ & $07 / 27 / 2016$ & NCT02857049 & $\begin{array}{l}\text { France, } \\
\text { Marseille }\end{array}$ & 220 & $\begin{array}{l}70 \text { years } \\
\text { and older }\end{array}$ & $\begin{array}{l}\text { Patient aged over } 70 \text { years, } \\
\text { hospitalized in a geriatric service for a } \\
\text { short stay; Patient malnourished or at } \\
\text { risk of malnutrition, justifying the } \\
\text { medical prescription of ONS; Patient } \\
\text { agreeing to participate in the study } \\
\text { and who signed the informed consent } \\
\text { form }\end{array}$ & $\begin{array}{l}\text { Oral nutritional } \\
\text { supplements (ONS) } \\
\text { degustation }\end{array}$ \\
\hline 6. & $\begin{array}{l}\text { Clinical } \\
\text { Trials.gov }\end{array}$ & $\begin{array}{l}\text { Efficacy of a High Quality Protein Leucine- } \\
\text { enriched Nutraceutical in Attenuating } \\
\text { Inactivity- and Hypo-energetic Diet- } \\
\text { induced Muscle Loss in Older Women }\end{array}$ & $06 / 20 / 2017$ & NCT03197402 & Canada & 20 & $\begin{array}{l}70 \text { to } 75 \\
\text { Years }\end{array}$ & $\begin{array}{l}\text { Females between } 70 \text { and } 75 \text { years of } \\
\text { age (a narrow age range improved } \\
\text { homogeneity of the sample for this } \\
\text { proof-of-concept trial) with a body } \\
\text { mass index (BMI) between } 27 \text { and } 35 \text {, } \\
\text { and muscle mass } 1 \text { standard deviation } \\
\text { below that of sex- and ethnicity- } \\
\text { matched } 30 \text { years old (note: this has } \\
\text { been called 'stage } 1 \text { sarcopenia' or } \\
\text { 'pre-sarcopenia') will be recruited. In } \\
\text { addition, participants will be non- } \\
\text { smokers and generally healthy per } \\
\text { responses to a standard health } \\
\text { screening questionnaire }\end{array}$ & $\begin{array}{l}\text { Dietary supplement: } \\
\text { Leucine-enriched protein } \\
\text { gel delivery system; } \\
\text { Dietary supplement: } \\
\text { Milk protein gel delivery } \\
\text { system } \\
\text { Non-leucine enriched }\end{array}$ \\
\hline
\end{tabular}




\begin{tabular}{|c|c|c|c|c|c|c|c|c|c|}
\hline No. & Register & Study title & $\begin{array}{l}\text { Date of } \\
\text { submission }\end{array}$ & ID-Nr. & Country & $\mathrm{n}$ & Age & Inclusion criteria & Intervention \\
\hline 7. & Clinical Trials.gov & $\begin{array}{l}\text { The Effects of Whey Versus Collagen } \\
\text { Protein Intake on Acute and Longer-term } \\
\text { Skeletal Muscle Protein Synthesis in } \\
\text { Older Adults }\end{array}$ & $09 / 11 / 2017$ & NCT03281434 & Canada & 24 & $\begin{array}{l}60 \text { to } 75 \\
\text { years }\end{array}$ & $\begin{array}{l}\text { healthy; ambulatory, and able to } \\
\text { perform exercise; active (3500- } \\
10000 \text { steps per day) }\end{array}$ & $\begin{array}{l}\text { Dietary supplement: } \\
\text { Collagen peptide. Low leucine } \\
\text { content protein supplement to be } \\
\text { consumed twice daily; } \\
\text { Dietary supplement: } \\
\text { Whey protein isolate. High quality } \\
\text { protein (DIAAS score), high } \\
\text { leucine supplement, to be } \\
\text { consumed twice daily }\end{array}$ \\
\hline 8. & Clinical Trials.gov & $\begin{array}{l}\text { Nutritional Strategies to Augment the } \\
\text { Postprandial Muscle Protein Synthetic } \\
\text { Response to the Ingestion of a Low Dose } \\
\text { of Protein in Middle-aged Women }\end{array}$ & $09 / 20 / 2016$ & NCT02918981 & USA & 60 & $\begin{array}{l}50 \text { to } 79 \\
\text { years }\end{array}$ & $\begin{array}{l}\text { BMl<30 kg/m2; healthy, } \\
\text { sedentary }\end{array}$ & $\begin{array}{l}\text { Behavioral: } \\
\text { Resistance Exercise. Unilateral leg } \\
\text { extension exercise immediately } \\
\text { prior to ingestion of the } \\
\text { experimental beverage. } \\
\text { Dietary supplement: } \\
14 \mathrm{~g} \text { Whey protein or: } 6.6 \mathrm{~g} \text { Whey } \\
\text { protein }+1.25 \mathrm{~g} \text { leucine or: } 4 \mathrm{~g} \\
\text { Whey protein }+1.25 \mathrm{~g} \text { leucine }+ \\
2.6 \mathrm{~g} \text { Whey peptides or: } 6.6 \mathrm{~g} \\
\text { Whey protein }+1.25 \mathrm{~g} \text { leucine }+ \\
0.8 \mathrm{~g} \text { Citrulline or: other: water } \\
\text { (control) }\end{array}$ \\
\hline
\end{tabular}




\begin{tabular}{|c|c|c|c|c|c|c|c|c|c|}
\hline No. & Register & Study title & Date of submission & ID-Nr. & Country & $\mathrm{n}$ & Age & Inclusion criteria & Intervention \\
\hline 9. & $\begin{array}{l}\text { Clinical } \\
\text { Trials.gov }\end{array}$ & $\begin{array}{l}\text { Effects of } 12 \\
\text { Weeks } \\
\text { Resistance } \\
\text { Exercise and } \\
\text { Whey Protein } \\
\text { Supplementation } \\
\text { on Multiple } \\
\text { Indices of } \\
\text { Sarcopenia in } \\
\text { Older Men }\end{array}$ & $09 / 11 / 2017$ & NCT03299972 & USA & 52 & $\begin{array}{l}60 \text { to } 80 \\
\text { years }\end{array}$ & $\begin{array}{l}\text { Retired Men aged } 60-80 \\
\text { years; Body Mass Index } \\
18.5-30 \mathrm{~kg} / \mathrm{m} 2 ; \text { Not } \\
\text { participated in } \\
\text { resistance exercise } \\
\text { within the last } 6 \\
\text { months; Free from } \\
\text { musculoskeletal injury }\end{array}$ & $\begin{array}{l}\text { Dietary Supplement: } \\
\text { Whey Protein } \\
25 \mathrm{~g} \text { of high leucine whey } \\
\text { protein supplementation } \\
\text { twice daily (breakfast } \\
\text { and lunch) } \\
\text { or: Placebo } \\
23.75 \mathrm{~g} \text { of placebo } \\
\text { powder twice daily } \\
\text { (breakfast and lunch) } \\
\text { or: Resistance Exercise + } \\
\text { Whey protein: } \\
60 \text { minutes of resistance } \\
\text { exercise twice a week } \\
\text { and } 25 \mathrm{~g} \text { of high leucine } \\
\text { whey protein twice daily; } \\
\text { or: Resistance exercise + } \\
\text { Placebo: } 60 \text { minutes of } \\
\text { resistance exercise twice } \\
\text { a week and } 23,75 \mathrm{~g} \text { of } \\
\text { placebo powder twice } \\
\text { daily }\end{array}$ \\
\hline 10. & $\begin{array}{l}\text { Clinical } \\
\text { Trials.gov }\end{array}$ & $\begin{array}{l}\text { Evaluation of a } \\
\text { High Calorie, } \\
\text { High Protein } \\
\text { Tube Feeding } \\
\text { Product in an } \\
\text { Adult Population } \\
\text { with or at Risk of } \\
\text { Malnutrition }\end{array}$ & $03 / 08 / 2018$ & NCT03459001 & Spain & 35 & $\begin{array}{l}\text { Adults } \\
\text { and } \\
\text { Seniors }\end{array}$ & $\begin{array}{l}\text { MUST score } \geq 2 \text {; Under } \\
\text { the care of a health care } \\
\text { professional for } \\
\text { malnutrition or at risk } \\
\text { of malnutrition and has } \\
\text { been prescribed the } \\
\text { study formula; Requires } \\
\text { and agrees to be tube } \\
\text { fed the enteral feeding } \\
\text { product for at least } 16 \\
\text { consecutive days as } \\
\text { their sole-source of } \\
\text { nutritional intake; Free } \\
\text { living in the community } \\
\text { or is residing in a } \\
\text { nursing home and } \\
\text { conforms to the } \\
\text { requirements set forth } \\
\text { on the study product } \\
\text { label. }\end{array}$ & $\begin{array}{l}\text { high calorie, high protein } \\
\text { tube feed }\end{array}$ \\
\hline
\end{tabular}




\begin{tabular}{|c|c|c|c|c|c|c|c|c|c|}
\hline No. & Register & Study title & $\begin{array}{l}\text { Date of } \\
\text { submission }\end{array}$ & ID-Nr. & Country & $\mathrm{n}$ & Age & Inclusion criteria & Intervention \\
\hline 11. & $\begin{array}{l}\text { Clinical } \\
\text { Trials.gov }\end{array}$ & $\begin{array}{l}\text { Sarcopenia Prevention with a Targeted Exercise and } \\
\text { Protein Supplementation Program (STRONG) }\end{array}$ & 0173172018 & NCT03417531 & Switzerland & 800 & $>75$ years & $\begin{array}{l}\text { At least } 1 \text { of } 5 \text { frailty } \\
\text { criteria, definition by } \\
\text { Linda Fried; low trauma } \\
\text { fall in the last } 12 \\
\text { months prior to } \\
\text { enrollment; Mini } \\
\text { Nutritional Assessment } \\
\text { (MNA) screening tool } \\
\text { (score } \leq 11 \text { ); } \\
\text { Community-dwelling or } \\
\text { assisted living }\end{array}$ & $\begin{array}{l}\text { L-leucine-enriched whey } \\
\text { protein isolate powder } \\
\text { (equivalent to } 20 \mathrm{~g} \text { of } \\
\text { Protein); twice daily; all } \\
\text { participants will receive a } \\
\text { control dose of } 24^{\prime} 000 \text { IU } \\
\text { vitamin D per month } \\
\text { home exercise strength } \\
\text { or flexibility ( } 3 \times 30 \\
\text { minutes/week) }\end{array}$ \\
\hline 12. & $\begin{array}{l}\text { Clinical } \\
\text { Trials.gov }\end{array}$ & $\begin{array}{l}\text { Development of a Decision Support System to Prevent } \\
\text { and Treat Disease-related Malnutrition }\end{array}$ & $01 / 10 / 2018$ & NCT03412695 & Norway & 100 & $\begin{array}{l}\text { Adults } \\
\text { and } \\
\text { Seniors }\end{array}$ & $\begin{array}{l}\text { At nutritional risk, } \\
\text { defined as Nutrition } \\
\text { Risk Screening (NRS- } \\
\text { 2002) score >3 }\end{array}$ & $\begin{array}{l}\text { nutritional treatment of } \\
\text { the patients } \\
\text { decision support system } \\
\text { "MyFood" are developed } \\
\text { in the project with the } \\
\text { purpose to prevent and } \\
\text { treat disease-related } \\
\text { malnutrition. }\end{array}$ \\
\hline 13. & $\begin{array}{l}\text { Clinical } \\
\text { Trials.gov }\end{array}$ & $\begin{array}{l}\text { Nutritional Perihabilitation in Older Veterans Undergoing } \\
\text { Surgery }\end{array}$ & $03 / 13 / 2018$ & NCT03073811 & USA & 105 & $>65$ years & $\begin{array}{l}\text { Elective abdominal } \\
\text { surgery; Referred to the } \\
\text { VA Perioperative } \\
\text { Optimization of Senior } \\
\text { Health Clinic; Able to } \\
\text { record dietary intake or } \\
\text { has a proxy who can } \\
\text { record dietary intake }\end{array}$ & $\begin{array}{l}30 \text { grams of high quality } \\
\text { protein ( } 20 \text { grams from } \\
\text { Ensure Enlive and } 10 \\
\text { grams of whey protein } \\
\text { powder) } \\
\text { educational on the role } \\
\text { of nutrition; Registered } \\
\text { Dietitian will instruct } \\
\text { participant to follow } \\
\text { instruction on handout }\end{array}$ \\
\hline 14. & $\begin{array}{l}\text { Clinical } \\
\text { Trials.gov }\end{array}$ & $\begin{array}{l}\text { Nutrition in Disguise: Development and Palatability } \\
\text { Testing of Novel Food Products (NiD) }\end{array}$ & $01 / 17 / 2018$ & NCT03402061 & Canada & 200 & $>65$ years & $\begin{array}{l}\text { Older adults living in the } \\
\text { community with no } \\
\text { food allergies; older } \\
\text { adults living in long } \\
\text { term care with and } \\
\text { without dementia with } \\
\text { no food allergies }\end{array}$ & $\begin{array}{l}\text { Dietary Supplement: } \\
\text { nutrient enhanced } \\
\text { recipes } \\
\text { Behavioral interventions }\end{array}$ \\
\hline 15. & $\begin{array}{l}\text { Clinical } \\
\text { Trials.gov }\end{array}$ & $\begin{array}{l}\text { Effects of Nutritional Supplementation and Dietary } \\
\text { Advice in Elderly Patients After Hospital Discharge. }\end{array}$ & $01 / 28 / 2010$ & NCT01057914 & Sweden & 800 & $\begin{array}{l}65 \text { years } \\
\text { and older }\end{array}$ & $\begin{array}{l}\geq 65 \text { years old; Admitted } \\
\text { to one of the } \\
\text { participating wards } \\
\text { during the recruitment } \\
\text { period }\end{array}$ & $\begin{array}{l}400 \text { kcal from a liquid } \\
\text { supplementation } \\
\text { formula } \\
\text { dietary advice for one } \\
\text { hour during the hospital } \\
\text { stay }\end{array}$ \\
\hline
\end{tabular}




\begin{tabular}{|c|c|c|c|c|c|c|c|c|c|}
\hline No. & Register & Study title & $\begin{array}{l}\text { Date of } \\
\text { submission }\end{array}$ & ID-Nr. & Country & $n$ & Age & Inclusion criteria & Intervention \\
\hline 16. & $\begin{array}{l}\text { WHO int. } \\
\text { Clinical trials } \\
\text { registry } \\
\text { platform }\end{array}$ & $\begin{array}{l}\text { Effectiveness of a combined } \\
\text { resistance exercise and nutrition } \\
\text { intervention to promote } \\
\text { maintenance of physical } \\
\text { functioning of community- } \\
\text { dwelling elderly in a real-life } \\
\text { setting }\end{array}$ & 05.09 .16 & NTR6038 & $\begin{array}{l}\text { Netherlands - Wageningen } \\
\text { University (WUR); sponsored by: } \\
\text { Ministry of Economic Affairs, } \\
\text { FrieslandCampina Innopastry }\end{array}$ & 200 & $\begin{array}{l}65 \text { years } \\
\text { or over }\end{array}$ & $\begin{array}{l}\text { Living independently in one of } \\
\text { the five selected } \\
\text { municipalities; able to } \\
\text { understand dutch language; } \\
\text { pre-frail or frail according to } \\
\text { fried criteria; having signed } \\
\text { informed consent }\end{array}$ & $\begin{array}{l}12 \text { weeks intervention: } \\
\text { 1) Twice weekly 1-Hour } \\
\text { progressive resistance-type } \\
\text { exercise training, in groups, } \\
\text { supervised by } \\
\text { physiotherapists } \\
\text { 2) Increased dietary protein } \\
\text { intake during the main } \\
\text { meals, using protein-rich } \\
\text { products, supervised by a } \\
\text { dietitian. } 12 \text { weeks } \\
\text { maintenance period: } \\
\text { participants will be } \\
\text { introduced to local exercise } \\
\text { and nutrition facilities. } \\
\text { Multiple activities will be } \\
\text { organized to stimulate } \\
\text { participants to maintain the } \\
\text { active lifestyle and an } \\
\text { adequate protein } \\
\text { consumption. } \\
\text { The control group receives } \\
\text { no treatment in the first } 24 \\
\text { weeks, and will receive the } \\
\text { maintenance period after } 24 \\
\text { weeks }\end{array}$ \\
\hline
\end{tabular}




\begin{tabular}{|c|c|c|c|c|c|c|c|c|c|}
\hline No. & Register & Study title & $\begin{array}{l}\text { Date of } \\
\text { submission }\end{array}$ & ID-Nr. & Country & $\mathrm{n}$ & Age & Inclusion criteria & Intervention \\
\hline 17. & $\begin{array}{l}\text { WHO int. } \\
\text { Clinical } \\
\text { trials } \\
\text { registry } \\
\text { platform }\end{array}$ & $\begin{array}{l}\text { PILOT STUDY } 2 \\
\text { ¡Prevention Of }^{\oplus \text { Malnutrition In Senior }} \\
\text { Subjects (PROMISS)i }\end{array}$ & $11 / 24 / 2017$ & NTR6849 & Netherlands & 100 & $>70$ years & $\begin{array}{l}\text { Community-dwelling; Lower } \\
\text { protein intake }(<1.0 \\
\mathrm{g} / \mathrm{kg} / \text { body weight) based on } \\
\text { a short food list that is } \\
\text { developed and validated } \\
\text { using an extended FFQ } \\
\text { among Dutch older adults } \\
\text { (data available on request); } \\
\text { Able to eat independently; } \\
\text { Willing to eat our provided } \\
\text { products; Able to speak, } \\
\text { write and read the Dutch } \\
\text { language }\end{array}$ & $\begin{array}{l}\text { Increased dietary } \\
\text { protein intake during } \\
\text { the main meals, using } \\
\text { protein-rich products, } \\
\text { supervised by a dietitian } \\
\text { yes; } 1 \text { ) replacing } \\
\text { habitual products with } \\
\text { the newly developed } \\
\text { protein rich food } \\
\text { products; and 2) } \\
\text { consumption of the } \\
\text { newly developed } \\
\text { protein rich food } \\
\text { products in addition to } \\
\text { their regular diet in } \\
\text { close proximity of } \\
\text { regular physical activity. }\end{array}$ \\
\hline 18. & anzctr & $\begin{array}{l}\text { Dietary protein } \\
\text { digestion and } \\
\text { absorption kinetics and } \\
\text { subsequent } \\
\text { postprandial muscle } \\
\text { protein accretion in } \\
\text { healthy older people }\end{array}$ & 09/25/2015 & $\begin{array}{l}\text { ACTRN126150011345 } \\
16\end{array}$ & $\begin{array}{l}\text { Australia - University of } \\
\text { Adelaide }\end{array}$ & 20 & $\begin{array}{l}65 \text { years and } \\
\text { older }\end{array}$ & $\begin{array}{l}\text { Male; Body Mass Index } \\
\text { (BMI): } 22-30 \mathrm{~kg} / \mathrm{m} 2 ; \text { Weight } \\
\text { stable ( }<5 \% \text { fluctuation in } \\
\text { body weight in previous } 3 \\
\text { months) }\end{array}$ & $\begin{array}{l}\text { Intraduodenal protein } \\
\text { infusion; } \\
\text { or: intraduodenal } \\
\text { administration of milk } \\
\text { protein (intrinsically } \\
\text { labelled with L- } \\
\text { Phenylalanine or L- } \\
\text { Leucine or ii) } \\
\text { or: amino acid mixture } \\
\text { Matching (isocaloric) } \\
\text { the milk protein } \\
\text { including L- } \\
\text { phenylalanine and L- } \\
\text { leucine }\end{array}$ \\
\hline
\end{tabular}




\begin{tabular}{|c|c|c|c|c|c|c|c|c|c|}
\hline No. & Register & Study title & $\begin{array}{l}\text { Date of } \\
\text { submission }\end{array}$ & ID-Nr. & Country & $\mathrm{n}$ & Age & Inclusion criteria & Intervention \\
\hline 19. & $\begin{array}{l}\text { EU Clinical } \\
\text { Trials } \\
\text { Register }\end{array}$ & $\begin{array}{l}\text { Safety and efficacy of subcutaneous (SC) } \\
\text { administration of Clinimix N9G15E in } \\
\text { elderly patients at risk for malnutrition, } \\
\text { at a dose of } 1 \text { liter infused over } 12 \text { hours } \\
\text { for } 7 \text { to } 10 \text { consecutive days. A } \\
\text { prospective, multicentre, randomized, } \\
\text { open-label, non-inferiority, controlled } \\
\text { phase III B trial carried out in parallel } \\
\text { groups: subcutaneous versus peripheral } \\
\text { intravenous administration }\end{array}$ & $04 / 23 / 2008$ & $\begin{array}{l}\text { EudraCT } \\
\text { Number: 2007- } \\
\text { 006153-31 }\end{array}$ & France & 126 & $\begin{array}{l}\text { Adults, } \\
\text { Elderly }\end{array}$ & $\begin{array}{l}\text { 1. Both genders } \\
\text { 2. Age superior or equal to } 65 \text { years } \\
\text { 3. Hospitalized and at risk for } \\
\text { malnutrition defined by their MNA } \\
\text { SF below } 12 \text { and GNRI between } \\
83.5 \text { and } 97.5 \text {. } \\
\text { 4. Oral intake }<50 \% \text { of appropriate } \\
\text { intake (defined as } 30 \mathrm{Kcal} / \mathrm{kg} / \text { day) } \\
\text { for an expected duration of } \\
\text { greater than } 3 \text { days. Severe } \\
\text { dehydrated patients must be } \\
\text { rehydrated before enrolling into } \\
\text { the trial } \\
\text { 5. Dated and signed written } \\
\text { informed consent, prior to any } \\
\text { study-related procedures, } \\
\text { provided by the patient or, } \\
\text { according to the law (L. } 1122-2 \text { ), } \\
\text { by his legal representative or by a } \\
\text { "personne de confiance" if any } \\
\text { written designation or, if not, his } \\
\text { family member or, if no family } \\
\text { member, a close relative } \\
\text { 6. Patients in stable condition }\end{array}$ & $\begin{array}{l}\text { Clinimix N9G15E at a dose } \\
\text { of } 1 \text { liter infused over } 12 \\
\text { hours for } 7 \text { to } 10 \\
\text { consecutive days }\end{array}$ \\
\hline 20. & $\begin{array}{l}\text { BioMed } \\
\text { Central } \\
\text { isrctn.com }\end{array}$ & $\begin{array}{l}\text { protein supplementation for preventing } \\
\text { bone loss and improving muscle strength } \\
\text { in older women: a randomized controlled } \\
\text { trial }\end{array}$ & $07 / 03 / 2017$ & ISRCTN12419961 & $\begin{array}{l}\text { United } \\
\text { Kingdom }\end{array}$ & 60 & $\begin{array}{l}65 \text { years } \\
\text { and older }\end{array}$ & $\begin{array}{l}\text { Community-dwelling women aged } \\
65 \text { or over; Not currently engaged } \\
\text { in upper body exercise training } \\
\text { (within last } 6 \text { months); Not using } \\
\text { any medications for osteoporosis } \\
\text { and not using any medications } \\
\text { that can affect bone metabolism; } \\
\text { Do not have an underlying disease } \\
\text { that impairs bone metabolism, } \\
\text { such as } \\
\text { hyper/hypoparathyroidism }\end{array}$ & $\begin{array}{l}\text { Group A: Home-based } \\
\text { exercise intervention } \\
\text { Group B: Home-based } \\
\text { exercise intervention and } \\
\text { leucine and vitamin D } \\
\text { enriched supplement } \\
\text { Group C: No intervention } \\
\text { (no treatment) }\end{array}$ \\
\hline
\end{tabular}




\section{Results}

We compiled a list of 20 intervention studies (see table 2 and figure 1). The list contains information about registry name, date of submission, ID-number, country, title, estimated participants, ages, inclusion criteria and types of intervention (energy-/protein intake, behavioral). In a more detailed excel sheet further information are listed e.g. duration, study start and status of recruiting participants. For further information, please contact dliesegang@nutrition.uni-kiel.de.

Most of the studies were found at ClinicalTrials.gov $(n=15)$ but we also found intervention studies at WHO int. Clinical trials registry platform, EU Clinical Trials Register, Australian New Zealand Clinical Trials Registry, and International Standard Randomized Controlled Trial Number Registry.
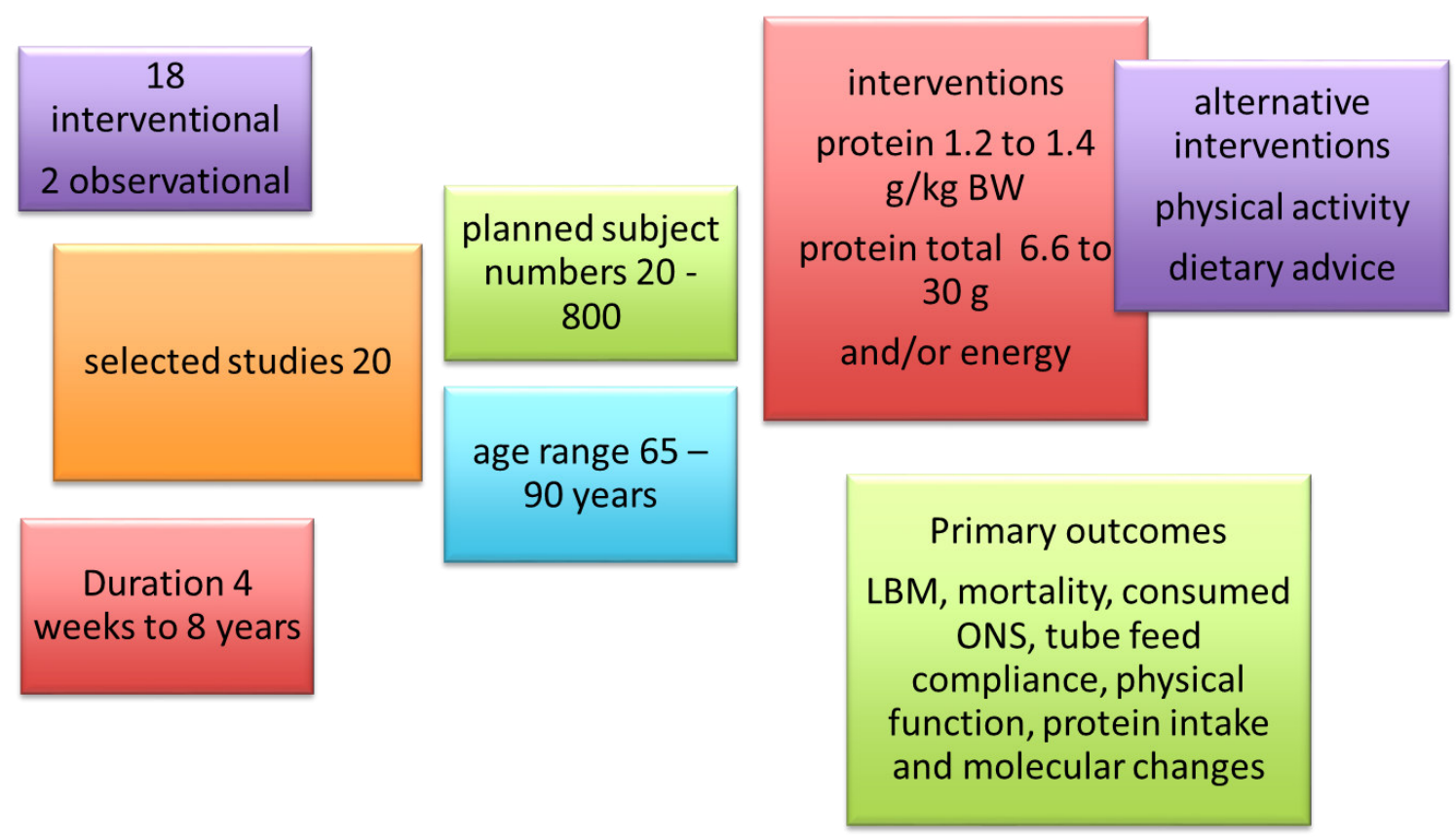

Figure 1 Overview of ongoing and planned non-pharmacological intervention studies

\section{Summary}

Most used screening tools in selected studies were:

1. Malnutrition: as inclusion criteria MNA (Mini Nutritional Assessment 2 studies), NRS-2002 (Nutritional Risk Screening 1 study) and ONS prescription (Oral Nutritional Supplements 2 studies)

2. Interventions with ONS: in three studies ONS only and in one plus dietary advice

3. behavioral interventions: in nine studies different behavioral interventions were included e.g. resistance training or dietary advice

4. Primary outcomes: for physical function SPPB (Short Physical Performance Battery 5 studies), SGA (Subjective Global Assessment 3 studies), for nutrition NRS-2002 (1 study), ADL (Activity of Daily Living), TUG (Timed Up and Go), HGS (Hand Grip Strength) and walking/gait speed 


\section{Acknowledgements}

This work was initiated by the Joint Programming Initiative A Healthy Diet for a Healthy Life. The funding agencies supporting the MaNuEL Knowledge Hub are as follows (in alphabetical order of participating Member State): Austria, Federal Ministry of Science, Research and Economy (BMWFW); France, Ecole Superieure d'Agricultires (ESA); Germany, Federal Ministry of Food and Agriculture (BMEL) represented by Federal Office for Agriculture and Food (BLE); Ireland, Department of Agriculture, Food and the Marine (DAFM) and the Health Research Board (HRB); Spain, Instituto de Salud Carlos III, and the SENATOR trial (FP7-HEALTH-2012-305930); and The Netherlands, The Netherlands Organization for Health Research and Development (ZonMw).

Funding by Federal Ministry of Food and Agriculture: Finance Number 315-06.01-2815ERA12E No conflict of interest. 